LAWRENCE LIVERMORE NAT IO N A L LABORATORY
Modeling the Long-Term Isolation Performance of Natural and Engineered Geologic CO2 Storage Sites

James W. Johnson, John J. Nitao, Joesph P. Morris

July 29, 2004

7th International Conference on Greenhouse Gas Control Technologies

Vancouver, Canada

September 5, 2004 through September 9, 2004 
This document was prepared as an account of work sponsored by an agency of the United States Government. Neither the United States Government nor the University of California nor any of their employees, makes any warranty, express or implied, or assumes any legal liability or responsibility for the accuracy, completeness, or usefulness of any information, apparatus, product, or process disclosed, or represents that its use would not infringe privately owned rights. Reference herein to any specific commercial product, process, or service by trade name, trademark, manufacturer, or otherwise, does not necessarily constitute or imply its endorsement, recommendation, or favoring by the United States Government or the University of California. The views and opinions of authors expressed herein do not necessarily state or reflect those of the United States Government or the University of California, and shall not be used for advertising or product endorsement purposes. 


\title{
MODELING THE LONG-TERM ISOLATION PERFORMANCE OF NATURAL AND ENGINEERED GEOLOGIC $\mathrm{CO}_{2}$ STORAGE SITES
}

\author{
James W. Johnson", John J. Nitao, \& Joseph P. Morris \\ Lawrence Livermore National Laboratory, L-221, P.O. Box 808, Livermore, CA 94550, USA
}

\begin{abstract}
Long-term cap rock integrity represents the single most important constraint on the long-term isolation performance of natural and engineered geologic $\mathrm{CO}_{2}$ storage sites. $\mathrm{CO}_{2}$ influx that forms natural accumulations and $\mathrm{CO}_{2}$ injection for EOR/sequestration or saline-aquifer disposal both lead to concomitant geochemical alteration and geomechanical deformation of the cap rock, enhancing or degrading its seal integrity depending on the relative effectiveness of these interdependent processes. This evolution of cap-rock permeability can be assessed through reactive transport modeling, an advanced computational method based on mathematical models of the coupled physical and chemical processes catalyzed by the influx event. Using our reactive transport simulator (NUFT), supporting geochemical databases and software (SUPCRT92), and distinct-element geomechanical model (LDEC), we have shown that influx-triggered mineral dissolution/precipitation reactions within typical shale cap rocks continuously reduce microfrac apertures, while pressure and effective-stress evolution first rapidly increase then slowly constrict them. For a given shale composition, the extent of geochemical enhancement is nearly independent of key reservoir properties (permeability and lateral continuity) that distinguish saline aquifer and $\mathrm{EOR} /$ sequestration settings and $\mathrm{CO}_{2}$ influx parameters (rate, focality, and duration) that distinguish engineered disposal sites and natural accumulations, because these characteristics and parameters have negligible impact on mineral reaction rates. In contrast, the extent of geomechanical degradation is highly dependent on these reservoir properties and influx parameters, because they effectively dictate magnitude of the pressure perturbation. Specifically, initial geomechanical degradation has been shown inversely proportional to reservoir permeability and lateral continuity and proportional to influx rate. As a result, while the extent of geochemical alteration is nearly independent of filling mode, that of geomechanical deformation is significantly more pronounced during engineered injection. This discrepancy limits the extent to which natural $\mathrm{CO}_{2}$ reservoirs and engineered disposal sites can be considered analogous, and further suggests that the secure cap rock of a given natural $\mathrm{CO}_{2}$ accumulation may be incapable of providing an effective seal in the context of an engineered injection. A new conceptual framework reveals that ultimate counterbalancing of opposing geochemical and geomechanical effects is feasible, which suggests that shale cap rocks may evolve into effective hydrodynamic seals-in both natural and engineered storage sites - as a function of progressive geochemical alteration that attends some degree of initial $\mathrm{CO}_{2}$ leakage.
\end{abstract}

\section{Introduction}

The success of engineered geologic $\mathrm{CO}_{2}$ storage hinges on our ability to identify optimal sites and forecast their long-term security. This ability, in turn, relies upon predictive models for assessing the relative effectiveness of $\mathrm{CO}_{2}$ migration and sequestration processes (isolation performance) as a function of key target-formation and cap-rock properties (screening criteria). It also relies on detailed knowledge of naturally occurring $\mathrm{CO}_{2}$ reservoirs and clear understanding of the extent to which they represent natural analogs to engineered storage sites. Among key screening criteria, long-term cap rock integrity represents the single most important constraint on long-term isolation performance, and among predictive methodologies, the reactive transport modeling approach is uniquely well suited to quantify this fundamental constraint for both natural and engineered settings.

Our recent modeling work [1-2] has focused on applying our unique computational toolbox to address this central issue of long-term hydrodynamic seal capacity in geologic $\mathrm{CO}_{2}$ storage sites. In the present contribution, we review the methodology and fundamental results of this research; i.e., the reactive transport modeling approach, geochemical and geomechanical contributions to cap-rock permeability evolution during and after $\mathrm{CO}_{2}$ influx, and a new conceptual framework for evaluating the net impact on long-term cap rock integrity of these contributions.

*Corresponding author: Tel. (925) 423-7352, Fax. (925) 422-7438, Email: jwjohnson@llnl.gov 


\section{Methodology}

Reactive transport modeling is an advanced computational method for quantitatively predicting the long-term consequences of natural or engineered perturbations to the subsurface environment. The method is based on mathematical models of the integrated thermal, hydrological, geochemical, and geomechanical processes that redistribute mass and energy in response to the disequilibrium state imposed by perturbation events such as magmatic intrusion or $\mathrm{CO}_{2}$ influx. Traditionally, such models have been developed as separate entities and applied as such to address specific issues relevant their individual scope. The fundamental advance embodied in reactive transport modeling is its explicit integration of these conceptually distinct process models. In practice, however, present-day simulators address and couple various subsets of these models, while the ultimate simulation tool—one that implements and explicitly couples all of the relevant processes-remains on the horizon. We have developed a unique computational capability that integrates a state-of-the-art reactive transport simulator, NUFT [3], comprehensive supporting geochemical software and databases, SUPCRT92 [4], and a versatile distinct-element geomechanical model, LDEC [5]. The current method for one-way coupling between NUFT and LDEC represents our integrated model's key approximation. Specifically, the NUFT-LDEC interface facilitates mapping pressure evolution into the corresponding effective stress, fracture aperture, and permeability history; however, at present, this geomechanical-dependent evolution (LDEC) is not back-coupled into the multiphase flow and reactive transport model (NUFT). In lieu of such bi-directional coupling, the net impact on cap rock integrity of concomitant geochemical and geomechanical processes is evaluated using a new conceptual model $[2,6]$ that depicts geochemical counterbalancing of geomechanical aperture evolution as a function of diffusion distance and reaction progress.

\section{Results and Discussion}

Long-term enhancement or degradation of cap rock integrity hinges on the relative contributions of geochemical alteration, which tends to reduce microfrac apertures in typical shale, and geomechanical deformation, which widens them. We first describe the geochemical contribution, which has been evaluated within a full system analysis of coupled hydrological and geochemical processes for Sleipner-like settings [1,7], and then present the geomechanical contribution, which has been analyzed as a function of hydrological processes, key reservoir properties, and $\mathrm{CO}_{2}$ influx parameters [2,6]. Finally, we discuss the ultimate net effect on cap rock integrity of these opposing geochemical and geomechanical contributions for both natural and engineered storage scenarios [2,6].

\section{Geochemical alteration processes}

Chemical interaction of influx $\mathrm{CO}_{2}$ and shale cap rock is primarily localized to shale interfaces with the underlying reservoir (Figure 1A), but it also occurs along intra-shale microfracs that permit some degree of immiscible $\mathrm{CO}_{2}$ penetration. In our models [1,7], the Fe-Mg-rich clays that characterize typical shale mineralogies are represented by $\mathrm{Mg}$-Chlorite, Fe-Mg carbonates are represented by magnesite, and the coupled mineral dissolution/precipitation reaction that leads to magnesite deposition can be expressed as:

$$
\begin{gathered}
\mathrm{KAlSi}_{3} \mathrm{O}_{8}[\mathrm{~K}-\text { feld }]+2.5 \mathrm{Mg}_{5} \mathrm{Al}_{2} \mathrm{Si}_{3} \mathrm{O}_{10}(\mathrm{OH})_{8}[\mathrm{Mg} \text {-chl }]+12.5 \mathrm{CO}_{2}(\mathrm{aq}) \\
\mathrm{KAl}_{3} \mathrm{Si}_{3} \mathrm{O}_{10}(\mathrm{OH})_{2}[\text { musc }]+1.5 \mathrm{Al}_{2} \mathrm{Si}_{2} \mathrm{O}_{5}(\mathrm{OH})_{4}[\mathrm{kaol}]+12.5 \mathrm{MgCO}_{3}[\text { magnesite }]+4.5 \mathrm{SiO}_{2}[\text { silica }]+6 \mathrm{H}_{2} \mathrm{O} .
\end{gathered}
$$

Within the 25-m shale cap rock, magnesite precipitation is limited to the basal $10 \mathrm{~m}$ (represented by two 5-m layers of grid cells), where minimal immiscible $\mathrm{CO}_{2}$ penetration occurs. Such precipitation is virtually limited to the basal 5 -m layer, where near uniform magnesite concentration of 0.57 vol.\% is obtained by the close of the 10 -year prograde (active-injection) phase. During the retrograde (post-injection) phase, immiscible $\mathrm{CO}_{2}$ trapped beneath the cap rock continues to promote carbonate cementation in the basal 5-m layer, where near uniform magnesite concentration approaches 1.0 vol.\% after 20 years (Figure 1B). Kinetic reaction (1) proceeds to the right with an increase in solid-phase volume of $18.5 \%$ (magnesite accounting for 47 vol.\% of the product assemblage). After 20 years porosity and permeability of the 5 -m cap-rock base have been reduced by $8 \%$ and $22 \%$, respectively, by this process (Figure 1C), which upon hypothetical completion at 130 years would reduce initial porosity by half and initial permeability by an order of magnitude (Figure 1D), thereby significantly improving cap-rock integrity. A natural analog to reaction (1) has been documented in the Ladbroke Grove natural gas field, where postaccumulation $\mathrm{CO}_{2}$ influx has converted Fe-rich chlorite to Fe-rich dolomite (ankerite), kaolinite, and silica [8]. 

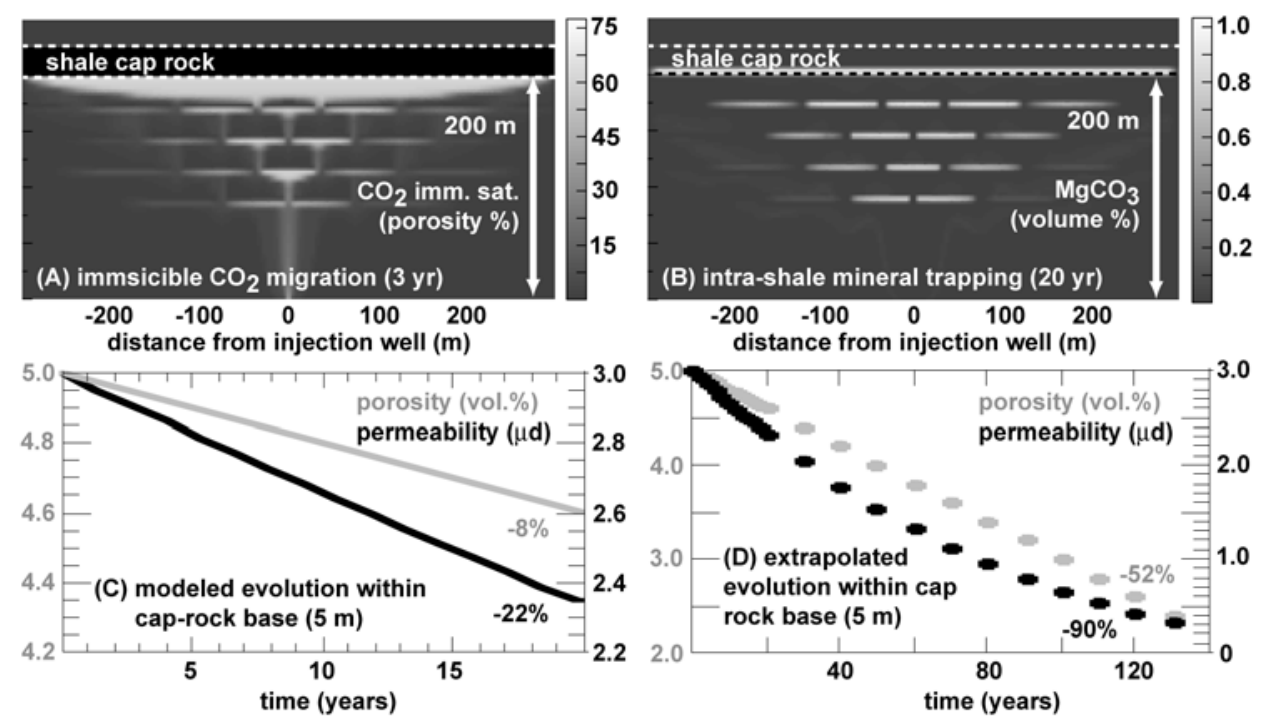

Figure 1. Immiscible $\mathrm{CO}_{2}$ migration (A), intra-shale mineral trapping (B), and porosity and permeability reduction in basal cap-rock shale (C-D) for Sleipner simulation DSH [1].

\section{Geomechanical deformation processes}

A first-order assessment of cap-rock geomechanical deformation can be obtained from evaluating the dependence of microfrac aperture evolution on the influx-triggered pressure perturbation. We have analyzed this dependence [2], first as a function of reservoir permeability and lateral continuity - two key parameters that typically distinguish saline-aquifer disposal sites and oil reservoirs, and second as a function of $\mathrm{CO}_{2}$ influx rate-the fundamental parameter that distinguishes engineered and natural storage scenarios.

\section{Dependence on reservoir properties: saline aquifer storage versus EOR settings}

Magnitude of the influx-triggered pressure perturbation within basal cap rock is inversely proportional to reservoir permeability and lateral continuity. For highly permeable, laterally extensive reservoirs (model UHP [2]), this perturbation follows a characteristic three-stage evolution: (1) rapid increase to maximum pressure as the aqueous phase is displaced upwards during initial plume ascent to the cap rock, (2) rapid asymptotic decrease to a near steady-state value between the ambient and maximum pressures that is maintained thereafter during the prograde regime, and (3) rapid asymptotic decrease towards the ambient value during the retrograde phase (Figures 2A-B). This pressure evolution suggests that the potential for dependent geomechanical deformation events is maximized during three very brief, distinct episodes that occur during the earliest stages of prograde and retrograde storage. Note that for this Sleipner-like setting, the range of injection-induced pressure variation is small: about 3 bars. Reservoir compartmentalization imposes two important variations on pressure evolution: (1) the magnitude of initial increase during plume ascent to the cap rock is significantly enhanced—reaching 60 bars in model CLP [2] — per restricted lateral flow of displaced formation water (Figures 2C-D), and (2) owing to presence of the accumulated $\mathrm{CO}_{2}$ column, during the retrograde phase pressure decays asymptotically toward a steady-state value that exceeds hydrostatic, thereby imposing a long-term pressure increase-about 5 bars in model CLP [2]—at and above the caprock/reservoir interface (Figure 2D).

Pressure evolution leads to geomechanical deformation of the cap rock through dependent changes in microfrac apertures. The functional form of aperture evolution within basal cap rock, evaluated using a simplified model for effective stress [2], is directly analogous to that described above for pressure, as exemplified by LDEC simulation of such evolution for model CLP (Figure 2D). Here, during the prograde phase apertures rapidly increase by roughly $1000 \mu \mathrm{m}$ during initial plume ascent, then asymptotically decrease to a value that reflects net widening of about 400 $\mu \mathrm{m}$. During the retrograde phase, they asymptotically decrease towards a value that reflects ultimate net widening of roughly $100 \mu \mathrm{m}$ per the 5-bar net pressure increase associated with $\mathrm{CO}_{2}$ accumulation. Unless counterbalanced by geochemical effects, this geomechanical widening could facilitate $\mathrm{CO}_{2}$ migration into the cap rock. 

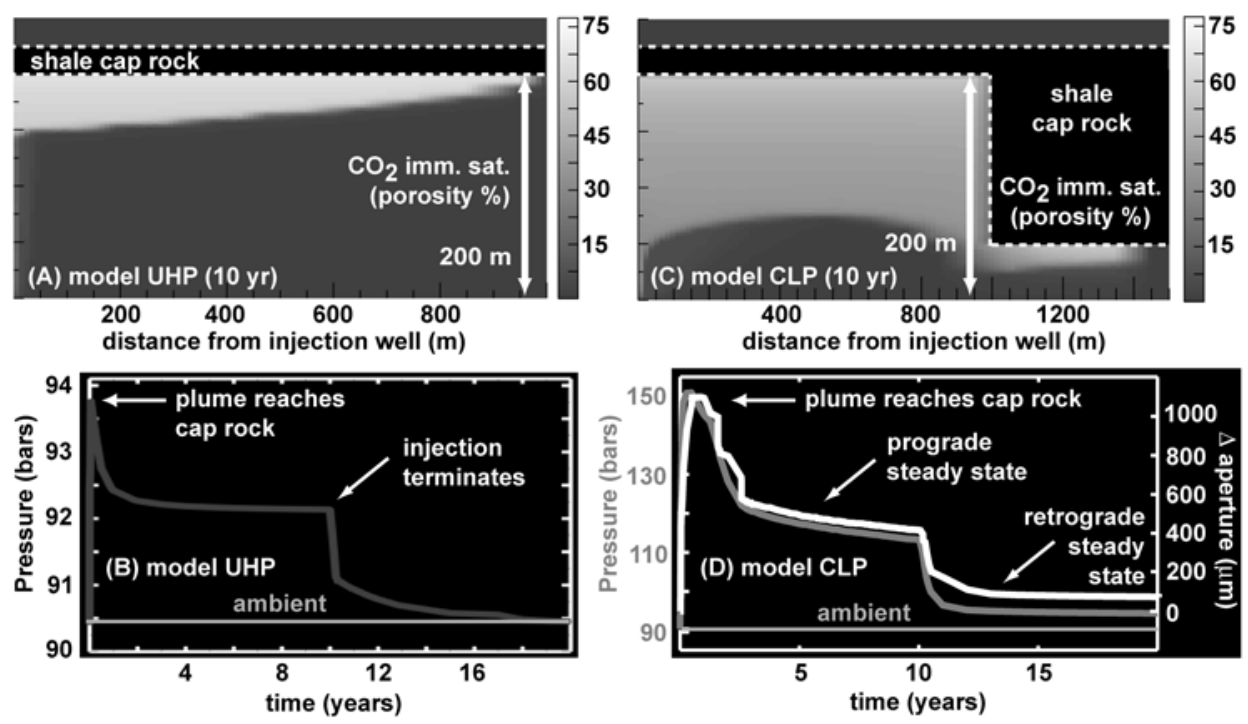

Figure 2. Immiscible $\mathrm{CO}_{2}$ migration within the reservoir and pressure history within the basal cap rock immediately above the injection point for models UHP (A-B) and CLP (C-D). Pressure dependent aperture evolution is shown for model CLP (D).

\section{Dependence on influx parameters: engineered versus natural storage}

Magnitude of the influx-triggered pressure perturbation within basal cap rock is proportional to $\mathrm{CO}_{2}$ influx rate. Here [2], this rate is varied from $10^{4}$ to $10^{3}$ to $10^{2}$ tons/yr, representing engineered injection and "fast" and "slow" natural accumulation scenarios, respectively, within a confined reservoir whose compartment width, height, and aspect ratio typify those of natural $\mathrm{CO}_{2}$ reservoirs [9]. The engineered injection rate is that used in the preceding simulations, while the adopted natural accumulation rates—which are presently unknown [9]—are rough estimates.

Because the engineered-injection model adopts the same injection rate used in the simulations described above, it illustrates dependence of the pressure perturbation on compartment width and aspect ratio, while providing a baseline for evaluating its dependence on influx rate. Increasing compartment width from 2 to $10 \mathrm{~km}$ causes pressure to increase even after the plume has reached the cap rock, owing to the increased volume of formation water that must be displaced. Hence, in this case pressure ultimately reaches 250 bars (perturbation of 160 bars) before declining after the plume reaches the lateral compartment boundary (cf. Figures 2C-D and 3A-B). When influx rate is reduced by one and two orders of magnitude, migration of the plume is retarded and the pressure perturbation is reduced proportionately. In the "fast" natural accumulation model, the plume does not reach the lateral compartment boundary until just before termination of the 40-year prograde event, while the maximum pressure perturbation (22 bars) is a factor of 7-8 less than that for the engineered injection model (Figure 3C-D). In the "slow" natural accumulation model, the plume has not quite advanced halfway to the compartment boundary after 60 years, while the maximum pressure perturbation is less than 3 bars. The extent of geomechanical cap-rock deformation through changes in effective stress and dependent aperture evolution is strongly dependent on influx rate: as the maximum pressure perturbation realized within basal cap rock increases from 3 to 22 to 160 bars with a 10- to 100-fold increase in influx rate, dependent aperture widening increases from 50 to 350 to $2900 \mu \mathrm{m}$ [2].

\section{Geomechanical counterbalancing of geochemical effects}

Ultimate enhancement or degradation of shale cap-rock integrity hinges on the relative effectiveness of geochemical and geomechanical processes. For a given dissolution/precipitation reaction within the matrix, the associated change in microfrac aperture $(\Delta \mathrm{a})$ depends on the initial volume fraction of the reactant assemblage $\left(\mathrm{V}_{\mathrm{R}} / \mathrm{V}_{\mathrm{T}}\right)$, standard molal volume change of the reaction $\left(\Delta \mathrm{V}_{\mathrm{r}}{ }^{0}=\mathrm{V}_{\mathrm{P}}{ }^{0}-\mathrm{V}_{\mathrm{R}}{ }^{\circ}\right)$, effective diffusion distance $\left(\mathrm{L}_{\mathrm{D}}\right)$, and reaction progress $(\mathrm{C})[2,6]$ :

$$
\Delta \mathrm{a}=-2\left[\left(\mathrm{~V}_{\mathrm{R}} / \mathrm{V}_{\mathrm{T}}\right)\left(\Delta \mathrm{V}_{\mathrm{r}}^{\mathrm{o}} / \mathrm{V}_{\mathrm{R}}^{0}\right) \mathrm{L}_{\mathrm{D}} \mathrm{C}\right] .
$$



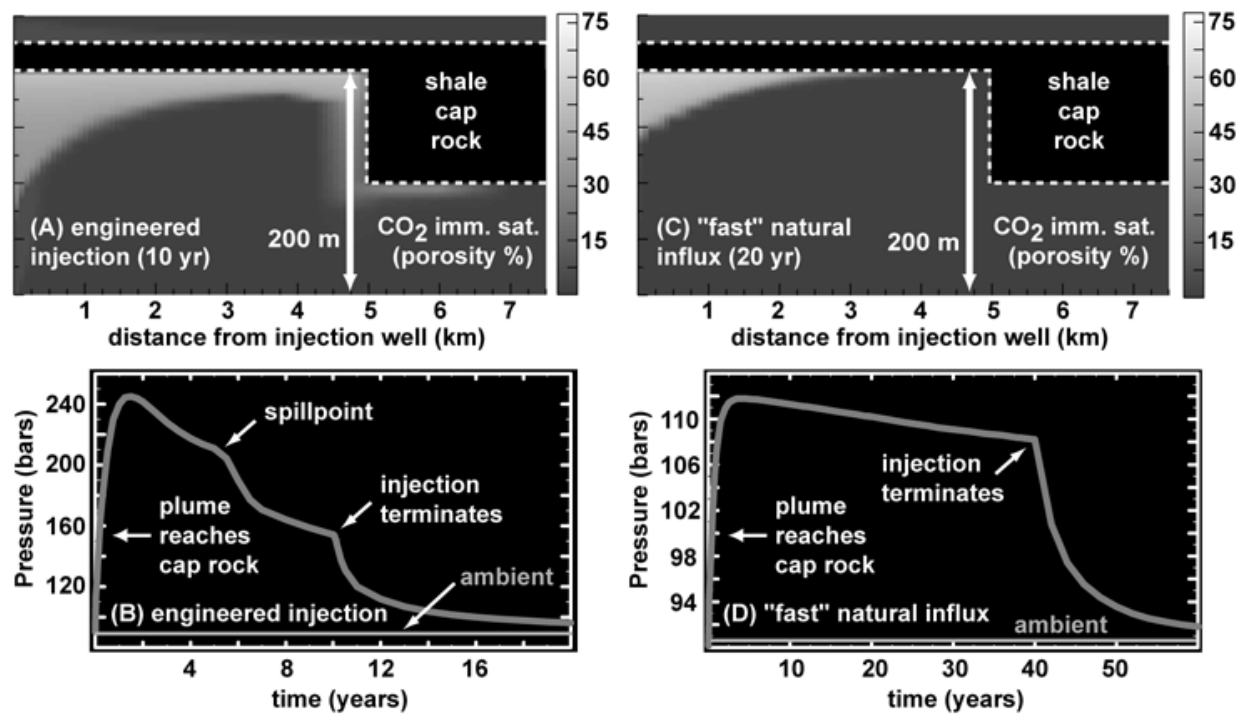

Figure 3. Immiscible $\mathrm{CO}_{2}$ migration within the reservoir and pressure history within the basal cap rock immediately above the injection point for distinct models representing engineered storage (A-B) and "fast" natural accumulation (C-D) scenarios.

All of these variables are typically known or can be closely estimated except for diffusion distance and reaction progress. Hence, it is both convenient and appropriate to plot $\Delta \mathrm{a}$ isopleths as a function of these latter two parameters. We have constructed such a diagram (Figure 4) for reaction (1), where the $\Delta \mathrm{a}$-isopleths plotted correspond to the range of geomechanical aperture widening-from initial maximum to final net values (roughly 1000 and $100 \mu \mathrm{m}$, respectively) — predicted for model CLP (Figure 2D). Hence, they can be viewed as geochemical counterbalance isopleths for this system; i.e., along any curve, departing to greater diffusion distances or reaction progress equates to net aperture closure (improved cap-rock integrity) as a function of combined geochemical and geomechanical effects, while departing to lesser values equates to net aperture opening (degraded integrity).

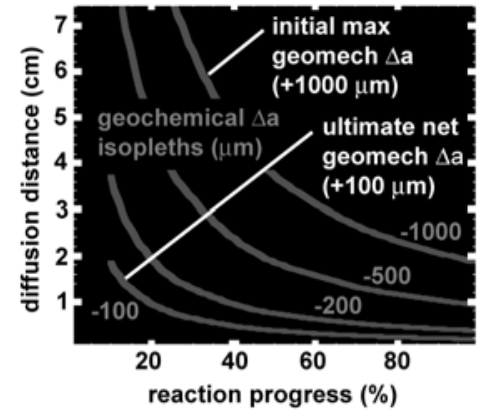

Figure 4. Conceptual framework for assessing potential long-term geochemical counterbalancing of geomechanical effects: geochemical $\Delta$ aperture isopleths plotted as a function of diffusion distance and reaction progress for mineral dissolution/ precipitation reaction (1), where $\Delta \mathrm{V}_{\mathrm{r}}{ }^{\circ} / \mathrm{V}_{\mathrm{R}}{ }^{\mathrm{o}}$ is 0.185 and $\mathrm{V}_{\mathrm{R}} / \mathrm{V}_{\mathrm{T}}$ is 0.1425 [1], constructed using equation (2) over the range of geomechanical $\Delta$ aperture defined by initial maximum and ultimate net widening for model CLP (Figure 2D).

This diagram reveals that ultimate geochemical counterbalancing of initial maximum aperture widening (1000 $\mu \mathrm{m}$ ) requires diffusion distances of 3-6.5 cm for reaction progress of 30-60\%. Moreover, such counterbalancing of the final net widening $(100 \mu \mathrm{m})$ requires $<0.5 \mathrm{~cm}$ diffusion distance for the same range of reaction progress; this diffusion length scale and extent of reaction progress - both of which are commonly observed in natural systemsstrongly suggest that $\mathrm{CO}_{2}$ influx-triggered geomechanical deformation may be ultimately counterbalanced by geochemical alteration. This raises the distinct possibility that currently secure shale cap rocks in natural $\mathrm{CO}_{2}$ reservoirs may have evolved into effective seals following some degree of $\mathrm{CO}_{2}$ migration into and perhaps through them. Careful mineralogical and petrographic analyses of such cap rocks may shed light on this important concept. 


\section{Conclusions}

For typical shale cap rocks, $\mathrm{CO}_{2}$ influx-triggered geochemical and geomechanical processes act in opposition to enhance and degrade hydrodynamic seal capacity though aperture narrowing and widening of microfracs. The extent of geochemical enhancement is largely independent of reservoir characteristics that distinguish saline-aquifer from EOR/sequestration settings and influx parameters that distinguish engineered disposal sites from natural accumulations, because such characteristics and parameters have negligible effect on mineral reaction rates [1]. In contrast, the extent of geomechanical degradation is highly dependent on these reservoir characteristics and influx parameters, because they effectively dictate magnitude of the pressure perturbation [2]. As a result, while the extent of geochemical alteration is nearly independent of filling mode, that of geomechanical deformation is significantly more pronounced during engineered storage. This suggests that the currently secure cap rock of a given natural $\mathrm{CO}_{2}$ accumulation may be incapable of providing an effective seal in the context of engineered injection. In addition, the pressure increase associated with $\mathrm{CO}_{2}$ accumulation in any compartmentalized system invariably results in net geomechanical aperture widening of cap-rock microfractures. This suggests that ultimate restoration of pre-influx hydrodynamic sealing capacity hinges on ultimate geochemical counterbalancing of this geomechanical effect, further suggesting that the well-documented leaky-to-secure character of fossil $\mathrm{CO}_{2}$ reservoirs may reflect the incomplete-to-complete nature of such restoration. A new conceptual framework [2,6] that depicts ultimate geochemical counterbalancing of geomechanical aperture evolution as a function of diffusion distance and reaction progress reveals diffusion length scales and reaction progress extents consistent with those observed in nature, which suggests that such counterbalancing is feasible; hence, shale cap rocks may in fact evolve into effective seals.

\section{Acknowledgements}

This work was performed under the auspices of the U.S. Department of Energy (US-DOE) by University of California, Lawrence Livermore National Laboratory under Contract W-7405-Eng-48. Funding support was provided by the JIP $\mathrm{CO}_{2}$ Capture Project and the US-DOE (Office of Fossil Energy) through a CRADA (TC-02038).

\section{References}

1. Johnson, J.W., J.J. Nitao, and K.G. Knauss, 2004, Reactive transport modeling of $\mathrm{CO}_{2}$ storage in saline aquifers to elucidate fundamental processes, trapping mechanisms, and sequestration partitioning: in Geol. Soc. London Spec. Pub. "Geologic Storage of Carbon Dioxide for Emissions Reduction: Technology”, Baines, S.J., and Worden, R.H., eds. (in press).

2. Johnson, J.W., J.J. Nitao, and J.P. Morris, 2004, Reactive transport modeling of cap rock integrity during natural and engineered $\mathrm{CO}_{2}$ storage: in "The $\mathrm{CO}_{2}$ Capture Project (CCP) for Carbon Dioxide Storage in Deep Geologic Formations for Climate Change Mitigation: Volume. 2 - Geologic Storage of Carbon Dioxide with Monitoring and Verification”, Benson, S.M., ed. (in press).

3. Nitao, J.J., 1998, Reference manual for the NUFT flow and transport code, version 2.0: Lawrence Livermore National Laboratory, UCRL-MA-130651, 55 p.

4. Johnson, J.W., E.H. Oelkers, and H.C. Helgeson, 1992, SUPCRT92: A software package for calculating the standard molal thermodynamic properties of minerals, gases, aqueous species, and reactions from 1 to 5000 bars and 0 to 1000C: Comp. Geosci., v. 18, n. 7, p. 899-947.

5. Morris, J.P., L.A. Glenn, and S.C. Blair, 2002, The distinct element method-application to structures in jointed rock: Lecture Notes Comp. Sci. Engr., v. 26, p. 291-306.

6. Johnson, J.W., J.J. Nitao, J.P. Morris, and S.C. Blair, 2003, Reactive transport modeling of geohazards associated with offshore $\mathrm{CO}_{2}$ injection for EOR and geologic sequestration: Proc. Offshore Technology Conf., Houston, TX, May 5-8, 2003, 9 p.

7. Johnson, J.W., and J.J. Nitao, 2002, Reactive transport modeling of geologic $\mathrm{CO}_{2}$ sequestration at Sleipner: Proc. Sixth Intl. Conf. Greenhouse Gas Control Tech. (GHGT-6), Kyoto, Japan, Oct 1-4, 2002, v. 1, p. $327-332$.

8. Watson, M.N., N. Zwingmann, and N.M. Lemon, 2002, The Ladbroke Grove-Katnook carbon dioxide natural laboratory: A recent $\mathrm{CO}_{2}$ accumulation in a lithic sandstone reservoir: Proc. Sixth Intl. Conf. Greenhouse Gas Control Technologies (GHGT-6), Kyoto, Japan, Oct 1-4, 2002, v. 1, p. 435-440.

9. Allis, R., T. Chidsey, W. Gywnn, C. Morgan, S. White, M. Adams, and J. Moore, 2001, Natural CO Reservoirs on the Colorado Plateau and Southern Rocky Mountains: Candidates for $\mathrm{CO}_{2}$ Sequestration: Proc. First Natl. Conf. Carbon Sequestration, Washington, DC, May 14-17, 2001, 19 p. 